\title{
Excited-State Molecular Dynamics Simulations of Conjugated Oligomers Using the Electronic Density Matrix
}

\author{
Matteo Tommasini, ${ }^{\dagger, \star}$ Giuseppe Zerbi, $\$$ Vladimir Chernyak, ${ }^{\S}$ and Shaul Mukamel ${ }^{*}, \dagger$ \\ Department of Chemistry, University of Rochester, Rochester, New York 14627, Dipartimento di Chimica \\ Industriale e Ingegneria Chimica, Politecnico di Milano, Piazza Leonardo da Vinci 32, 20133 Milano, Italy, \\ and Corning Incorporated, Process Engineering and Modeling, Corning, New York 14891
}

Received: January 23, 2001; In Final Form: April 12, 2001

\begin{abstract}
Vibrational motions in optically excited polyacetylene and polydiacetylene oligomers are calculated using potential surfaces obtained from the collective electronic oscillators (CEO) technique. The role of the effective conjugation coordinate (ECC) in the relaxation processes following an impulsive vertical excitation from the ground state is demonstrated. Real-space analysis of the electronic transition density matrices shows the charge and bond-order redistribution taking place upon photoexcitation.
\end{abstract}

\section{Introduction}

The major features of the vibrational Raman and infrared spectra of several families of conjugated molecules, such as polyacetylene and carotenoids, can be accounted for using a collective effective conjugation coordinate (ECC). ${ }^{1-9}$ In this paper, we present a simulation study of the ultrafast vibrational relaxation processes of polyenes following photoexcitation to an excited-state potential surface. The nature of the ECC coordinate and the mechanism for its excitation are demonstrated.

The ECC has been originally introduced to describe the Raman spectra of polyenes and polyacetylene. Out of the many vibrational degrees of freedom in large polyenes, only two intense bands around 1500 and $1150 \mathrm{~cm}^{-1}$ dominate the Raman spectra, and their intensity is larger by orders of magnitude than that of other Raman active bands. [The laser excitation in those experiments was $\lambda_{\text {exc }}=1064 \mathrm{~nm}$, below the energy gap which for polyacetylene is $1.4 \mathrm{eV}(890 \mathrm{~nm}) .^{12}$ ] For example, in transdodecahexaene, a medium-size polyacetylene oligomer, the ratio between the Raman intensity integrated over the entire $\mathrm{C}-\mathrm{H}$ stretching region $\left(2850-2950 \mathrm{~cm}^{-1}\right)$ and the strongest Raman band is about 10. Resonant Raman spectra generally show few lines (in most cases corresponding to totally symmetric vibrations) which are strongly coupled to the transition from the ground state to the excited state. ${ }^{10,11}$ The similarity of the resonant and the off-resonant Raman spectra of polyacetylene suggests that in this case it may be possible to describe the Raman process using a two-state model. The relevant vibrational coordinate connects the equilibrium geometry of the excited state with the equilibrium geometry of the ground state. The structural parameter most heavily modified by the lowest optical transition in polyenes is the degree of bond alternation along the chain. ${ }^{6-8}$ In particular, the double $\mathrm{C}=\mathrm{C}$ bonds become longer, while the single $\mathrm{C}-\mathrm{C}$ bonds become shorter. The ECC coordinate for

\footnotetext{
* Corresponding author.

University of Rochester.

$\doteqdot$ Politecnico di Milano.

$\S$ Corning Incorporated.
}

polyacetylene has therefore been defined as

$$
\mathrm{R}=\frac{r_{\mathrm{C}=\mathrm{C}}-R_{\mathrm{C}-\mathrm{C}}}{\sqrt{2}}
$$

Definitions of the ECC based on spectroscopic grounds were also proposed for other conjugated polymers (e.g., polythiophene, poly- $p$-phenylenevinylene).$^{5}$ For finite-size transpolyenes (such as carotenoids), the simple generalization of eq 1 is

$$
\mathrm{R}=\frac{\sum_{j=1, N_{\mathrm{C}=\mathrm{C}}} r_{j}-\sum_{j=1, N_{\mathrm{C}-\mathrm{C}}} R_{j}}{\sqrt{N_{\mathrm{C}=\mathrm{C}}+N_{\mathrm{C}-\mathrm{C}}}}
$$

The present study shows how to improve this definition by directly computing the nuclear relaxation of the molecule following a transition from the ground state to an excited state, thus introducing different weighting factors for each of the stretching coordinates. Definitions 1 and 2 assume that the variation of the equilibrium geometry induced by the photoexcitation mainly involves stretching degrees of freedom and that it can be approximated by a finite displacement $\Delta R$ along the ECC coordinate.

Conjugated molecules such as push-pull polyenes exhibit both a high Raman cross section and an intense infrared absorption for vibrational bands with high ECC character. A model based on this vibrational coordinate has been introduced to explain the relationship between the observed infrared and Raman intensities. ${ }^{9}$ The strong infrared intensity is related to high charge fluxes associated with the ECC of the $\pi$ system polarized by the donor-acceptor pair. The strong dopinginduced infrared bands of polyacetylene (IRAV) have been similarly attributed to large charge fluxes during ECC oscillations. $^{1}$

The ECC further successfully accounts for the magnitude of the vibrational contribution to the static hyperpolarizabilities of conjugated molecules. The electron-phonon coupling along 
this vibrational coordinate is strong, since the changes induced in the electronic wave function by this nuclear motion are most relevant for the optical response. ${ }^{13}$ When an electric field is applied to a molecule, the nuclei experience a force and are driven into a new equilibrium geometry. In the harmonic approximation, it is possible to calculate this geometry change and to derive from it closed expressions for the vibrational contributions to the molecular hyperpolarizabilities. ${ }^{14}$ It has been shown that in conjugated systems the vibrational contribution to the static hyperpolarizabilities which generally comes from all vibrational normal modes ${ }^{14}$ can be adequately approximated by the contribution of the ECC relaxation process ${ }^{15-17}$

$$
\begin{gathered}
\beta_{i j k}^{\mathrm{v}} \approx\left(\mathbf{F}^{-1}\right)_{\mathrm{R} R}\left(\frac{\partial \mu_{i}}{\partial \mathrm{R}} \frac{\partial \alpha_{j k}}{\partial \mathrm{R}}+\frac{\partial \mu_{j}}{\partial \mathrm{R}} \frac{\partial \alpha_{i k}}{\partial \mathrm{R}}+\frac{\partial \mu_{k}}{\partial \mathrm{R}} \frac{\partial \alpha_{i j}}{\partial \mathrm{R}}\right) \\
\gamma_{i j k l}^{\mathrm{v}} \approx\left(\mathbf{F}^{-1}\right)_{\mathrm{R} \mathrm{R}}\left(\frac{\partial \alpha_{i j}}{\partial \mathrm{R}} \frac{\partial \alpha_{k l}}{\partial \mathrm{R}}+\frac{\partial \alpha_{i k}}{\partial \mathrm{R}} \frac{\partial \alpha_{j l}}{\partial \mathrm{R}}+\frac{\partial \alpha_{i l}}{\partial \mathrm{R}} \frac{\partial \alpha_{j k}}{\partial \mathrm{R}}\right)+ \\
\left(\mathbf{F}^{-1}\right)_{\mathrm{R} \mathrm{R}}\left(\frac{\partial \mu_{i}}{\partial \mathrm{R}} \frac{\partial \beta_{j k l}}{\partial \mathrm{R}}+\frac{\partial \mu_{j}}{\partial \mathrm{R}} \frac{\partial \beta_{i k l}}{\partial \mathrm{R}}+\frac{\partial \mu_{k}}{\partial \mathrm{R}} \frac{\partial \beta_{i j l}}{\partial \mathrm{R}}+\frac{\partial \mu_{l}}{\partial \mathrm{R}} \frac{\partial \beta_{i j k}}{\partial \mathrm{R}}\right)
\end{gathered}
$$

The quantities $\partial \mu_{i} / \partial \mathrm{R}, \partial \alpha_{i j} / \partial \mathrm{R}$, and $\partial \beta_{\mathrm{jk} l} / \partial \mathrm{R}(i, j, k$, and $l$ indicate the Cartesian components) are respectively related to infrared intensities, Raman, and hyper-Raman cross sections of vibrations with high ECC character. $\left(\mathbf{F}^{-1}\right)_{\mathrm{R} R}$ represents the diagonal ECC component of the inverse harmonic force field.

We have developed a computer code MDCEOV (molecular dynamics CEO algorithm using a Verlet integrator; ${ }^{18}$ this program described in section II uses a molecular dynamics algorithm ${ }^{18}$ ) based on the electronic density matrix and on the collective electronic oscillators (CEO) description of molecular excitations. ${ }^{19-23}$ In section III, we demonstrate how analysis of the time evolution of the nuclear coordinates following an impulsive excitation shows clearly the features of the ECC coordinate discussed above for polyenes. It is possible to relate this coordinate to the time evolution of the ground- and the excited-state energy. Our simulations provide a direct firstprinciples test for the phenomenological ECC model; the ECC can be directly identified and characterized by monitoring the time evolution of the bond lengths. Computations performed on the polyene $\mathrm{C}_{10} \mathrm{H}_{12}$ with five $\mathrm{C}=\mathrm{C}$ bonds show that upon excitation the time evolution of the bond lengths involves the stretching of the double $\mathrm{C}=\mathrm{C}$ bonds, accompanied by the shrinking of the single $\mathrm{C}-\mathrm{C}$ bonds, as expected from the ECC model. We have further employed the same simulation algorithm to compute the vibrational mode couplings in polydiacetylene (PDA). Our calculations reveal strong interactions between stretching and bending vibrations, as observed in recent $5 \mathrm{fs}$ pump-probe measurements. ${ }^{24}$

\section{The Molecular Dynamics CEO Verlet Algorithm}

Simulations were carried out using a molecular dynamics method based on the collective electronic oscillators (CEO) approach, which generates excited-state surfaces while avoiding the direct computation of excited-state many-electron wave functions. Computation of excited-state wave functions is prohibitively expensive, particularly when it needs to be repeated for many nuclear configurations along a trajectory. The main bottleneck in molecular dynamics simulations of photoinduced processes is the computation of excited-state properties: energy surfaces, their gradients with respect to nuclear coordinates, and non adiabatic couplings. ${ }^{25}$ The CEO provides a fast, inexpensive algorithm for computing all of these quantities. It is based on a reduced description of the electronic structure which uses a minimal amount of relevant excited-state information and focuses on the time-dependent single-electron density matrix $\rho_{i j}(t) \equiv\left\langle\Psi(t)\left|c_{i}^{\dagger} c_{j}\right| \Psi(t)\right\rangle$ of the molecule driven by an external optical field $\epsilon(t)$. Here $\Psi(t)$ is the many-electron wave function, and $c_{i}^{\dagger}\left(c_{i}\right)$ 's are the fermionic creation (annihilation) operators for the atomic orbital $\chi_{i}$, which satisfy the Fermi anticommutation relationships

$$
\left[c_{i}^{\dagger}, c_{j}\right]_{+}=\delta_{i j} \quad\left[c_{i}^{\dagger}, c_{j}^{\dagger}\right]_{+}=0 \quad\left[c_{i}, c_{j}\right]_{+}=0
$$

The molecular Hamiltonian can be written as ${ }^{26}$

$$
\hat{H}=\sum_{i j} t_{i j} c_{i}^{\dagger} c_{j}+\frac{1}{2} \sum_{i j k l}\langle i j \mid k l\rangle c_{i}^{\dagger} c_{j}^{\dagger} c_{l} c_{k}-\sum_{s=x, y, z} \epsilon^{s}(t) \sum_{i j} \mu_{s i}^{j} c_{i}^{\dagger} c_{j}
$$

The single-electron matrix elements $t_{i j}$, Coulomb matrix elements $\langle i j \mid k l\rangle \equiv(i k \mid j l)$, and dipole moment matrix elements $\boldsymbol{\mu}_{i j}$ may be expressed in terms of the atomic orbitals $\chi_{i}(\mathbf{r})$

$$
\begin{gathered}
t_{i j}=\int \chi_{i}^{*}(\mathbf{r})\left[-\frac{1}{2} \nabla_{\mathbf{r}}^{2}+v_{\text {core }}(\mathbf{r})\right] \chi_{j}(\mathbf{r}) \mathrm{d} \mathbf{r} \\
(i j \mid k l)=\iint \frac{\chi_{i}^{*}\left(\mathbf{r}_{1}\right) \chi_{j}\left(\mathbf{r}_{1}\right) \chi_{k}^{*}\left(\mathbf{r}_{2}\right) \chi_{l}\left(\mathbf{r}_{\mathbf{2}}\right)}{\left|\mathbf{r}_{\mathbf{1}}-\mathbf{r}_{\mathbf{2}}\right|} \mathrm{d} \mathbf{r}_{\mathbf{1}} \mathrm{d} \mathbf{r}_{\mathbf{2}} \\
\boldsymbol{\mu}_{i j}=\int \chi_{i}^{*}(\mathbf{r}) \mathbf{r} \chi_{j}(\mathbf{r}) \mathrm{d} \mathbf{r}
\end{gathered}
$$

When the molecule is driven optically, its wave function $|\Psi(t)\rangle$ and consequently also the single-electron density matrix $\rho(t)=\bar{\rho}+\delta \rho(t)$ become time-dependent. Here $\bar{\rho}$ represents the ground-state density matrix, and the field-induced component $\delta \rho(t)$ is determined by solving the Heisenberg equations of motion for $c_{i}^{\dagger} c_{j}$. The many-body hierarchy is closed by invoking the time-dependent Hartree-Fock (TDHF) ansatz (i.e., assuming that $|\Psi(t)\rangle$ is represented by a single Slater determinant at all times), resulting in closed nonlinear equations of motion for $\delta \rho(t) .{ }^{20} \delta \rho(t)$ is further expanded in the eigenmodes $\xi_{v}$ of the effective Liouville operator $L(\mathbf{x})$ representing the linearized TDHF equations ( $\mathbf{x}$ denotes the set of nuclear coordinates $x_{\alpha}$ )

$$
\left.\left.L(\mathbf{x}) \mid \xi_{v}(\mathbf{x})\right)=\Omega_{v}(\mathbf{x}) \mid \xi_{v}(\mathbf{x})\right)
$$

The electronic normal modes $\left.\mid \xi_{v}(\mathbf{x})\right)$ correspond to the transition density matrices between the ground $|0\rangle$ and excited $|v\rangle$ states: $\left(\xi_{v}\right)_{i j} \equiv\left\langle v\left|c_{i}^{\dagger} c_{j}\right| 0\right\rangle$. The eigenvalues $\Omega_{v}(\mathbf{x})$ provide the excitation frequencies. Solving eq 9 at various nuclear configurations $\mathbf{x}$ yields the variation of the excitation energies $\Omega_{v}$ with $\mathbf{x}$. Thus, the excited-state adiabatic surface $E_{v}(\mathbf{x})$ can be computed by simply adding $\Omega_{v}(\mathbf{x})$ to the ground-state adiabatic surface $E_{0}(\mathbf{x})$

$$
E_{v}(\mathbf{x})=E_{0}(\mathbf{x})+\Omega_{v}(\mathbf{x})
$$

The ground-state energy is obtained directly from the HartreeFock ground-state density matrix

$$
E_{0}=2 \operatorname{Tr}\left[\bar{\rho} t+\frac{1}{2} \bar{\rho} V \bar{\rho}\right]
$$

The Liouville operator $L$ in eq 9 is given by

$$
L \xi=[F, \xi]+[V \xi, \bar{\rho}]
$$

$F$ is the Fock matrix, and $V$ is the operator representing the electron-electron interaction. For a closed shell system, $V$ is 
defined through the action of the Coulomb operator $J$ and the exchange operator $K$ on a generic matrix $\eta$ in the single electron space as follows:

$$
\begin{gathered}
(V \eta)_{i j}=2(J \eta)_{i j}-(K \eta)_{i j} \\
(J \eta)_{i j}=\sum_{i j}(i j \mid k l) \eta_{k l} \\
(K \eta)_{i j}=\sum_{i j}(i k \mid j l) \eta_{k l}
\end{gathered}
$$

The elements of the Fock matrix are

$$
F_{i j}=t_{i j}+(V \bar{\rho})_{i j}
$$

Vibronic coupling enters eq 9 through the dependence of the electronic Hamiltonian matrix elements $t_{i j}$, $(i j \mid k l)$, and thus $L$ on nuclear geometry.

In regions of nuclear configuration space far from avoided crossing or conical intersection points, the nuclear degrees of freedom can be usually treated classically and satisfy Newton's equation of motion

$$
m_{\alpha} \frac{\mathrm{d}^{2} x_{\alpha}}{\mathrm{d} t^{2}}=-\frac{\mathrm{d}}{\mathrm{d} x_{\alpha}}\left[E_{0}(\mathbf{x})+\Omega_{v}(\mathbf{x})\right]
$$

Vibrational dynamics thus requires the gradients of the excitedstate surfaces, representing the classical forces acting on the nuclei. These gradients can be computed through the derivative of eq 9 with respect to nuclear coordinates. As will be shown in the Appendix, this results in the expectation value of the derivative of the Liouville operator $L$ on a given excited state (we use a superscript $x$ to denote $\mathrm{d} / \mathrm{d} x$, and we drop the coordinate subscript $\alpha$ )

$$
\Omega_{v}^{x}=\left(\xi_{v}, L^{x} \xi_{v}\right)=\left(\xi_{v}\left|L^{x}\right| \xi_{v}\right)
$$

This form resembles the Hellmann-Feynman theorem for the computation of energy gradients. ${ }^{27}$ In eq 16 , we have introduced a scalar product between vectors in Liouville space

$$
\left(\eta_{1}, \eta_{2}\right)=\left(\eta_{1} \mid \eta_{2}\right)=\operatorname{Tr}\left(\bar{\rho}\left[\eta_{1}^{\dagger}, \eta_{2}\right]\right)
$$

With respect to this scalar product, the Liouville operator is hermitian: $\left(L \eta_{1}, \eta_{2}\right)=\left(\eta_{1}, L \eta_{2}\right)$.

It is possible to express this gradient as a finite difference (for sufficiently small $\Delta$ )

$$
\Omega_{v}^{x} \approx \frac{\left(\xi_{v}(x), L(x+\Delta) \xi_{v}(x)\right)-\left(\xi_{v}(x), L(x-\Delta) \xi_{v}(x)\right)}{2 \Delta}
$$

Finally, the gradient of the excited-state hypersurface $E_{v} \equiv E_{0}$ $+\Omega_{v}$ is given by

$$
\frac{\partial E_{v}}{\partial x_{\alpha}}=\frac{\partial E_{0}}{\partial x_{\alpha}}+\frac{\partial \Omega_{v}}{\partial x_{\alpha}}
$$

and the ground-state energy derivative can be expressed as

$$
E_{0}^{x}=2 \operatorname{Tr}\left[\bar{\rho} t^{x}+\frac{1}{2} \bar{\rho} V^{x} \bar{\rho}\right]
$$

The present computations were carried out using the MDCEOV program, which extends the CEO code, ${ }^{20,22,23,28}$ by implementing the computation of the gradients of the excitation energies and a Verlet-style molecular dynamics trajectory generator. ${ }^{18}$ The CEO code uses the ZINDO/S semiempirical spectroscopic Hamiltonian. Excited-state molecular dynamics computations require the CEO eigenvalues $\Omega_{v}$ and eigenvectors $\xi_{v}$ of the Liouville operator $L$ introduced previously. The Liouville operator is a superoperator acting in the space of single electron density matrices. It is a tetradic operator that depends on four indexes

$$
(L \xi)_{i j}=\sum_{k l} L_{i j k l} \xi_{k l}
$$

The matrix elements of $L$ can be calculated by using eqs 12 and 21. A transformation of indexes such as $\{i j\} \rightarrow N(i-1)+$ $j$ and $\{k l\} \rightarrow N(k-1)+l, N$ being the basis set size, brings $L_{i j k l}$ into a two indexes matrix $L_{\{i j\}\{k l\}}$ suitable for standard diagonalization algorithms. Since the number of elements in $L_{i j k l}$ grows with the fourth power of the basis set size, then except for small systems, it is generally not possible to handle the full matrix by conventional diagonalization approaches. To overcome this difficulty, we introduced a Lanczos algorithm for the computation of the low-lying eigenvalues of $L .{ }^{28}$ Usually, only a few eigenvalues are sufficient for the computation of the optical response. A strict convergence of the eigenvectors $\xi_{v}$ is required for a reliable computation of the excitation energy gradient, $\Omega_{v}^{x}=\left(\xi_{v}\left|L^{x}\right| \xi_{v}\right)$. To reduce the time requirements of the CEO Lanczos algorithm, we interfaced our code with the public domain ARPACK package. ${ }^{29}$ This library is especially designed to solve large eigenvalue problems and gives a set of well-converged orthogonal eigenvectors $\xi_{v}$. The basic idea behind both Lanczos and ARPACK algorithms is the iterated use of the definition of the action of $L$ on a vector $\eta$ to construct from a suitable first guess a sequence of vectors. These vectors span a Krylov subspace used to approximate the eigenvectors and eigenvalues. In the Lanczos algorithm, the Krylov space is used to approximate just one eigenvalue/eigenvector at a time. A sequence of eigenvalues is then generated through a deflation technique using the previously computed eigenvectors. In contrast, the ARPACK algorithm directly computes a set of eigenvalues and eigenvectors using a variant of the Arnoldi algorithm known as the implicitly restarted Arnoldi method. Implicit restarting provides a way to extract the important information from large Krylov subspaces, avoiding the storage and numerical difficulties associated with the standard Arnoldi approach. This is accomplished by continually compressing the meaningful information into a fixed size Krylov subspace. Details of this technique can be found in ref 29. Since the Liouville operator is not hermitian, special care needs to be taken when applying the Lanczos approach for the CEO. ${ }^{28}$ The ARPACK algorithm can handle general nonhermitian matrices such as $L$. Similar to the Lanczos algorithm, all it needs is to define the action of the Liouville operator $L$ on a generic vector $\eta$. Another attractive feature of the eigensolver ARPACK is the possibility of easily implementing the parallel version of the library, P_ARPACK, to substantially improve the performance. The ground-state energy surfaces and vibrational frequencies may be improved by using different semiempirical Hamiltonians such as AM1 and PM3, parametrized explicitly to reproduce experimental geometries. To integrate the classical equations of motion for the nuclear coordinates, we used a Verlet-type algorithm, commonly used in molecular dynamics simulations, ${ }^{18}$ which is more stable and efficient than a more general Runge-Kutta integration. In particular, the Verlet algorithm is known to conserve the total energy over a long time scale..$^{30}$ 

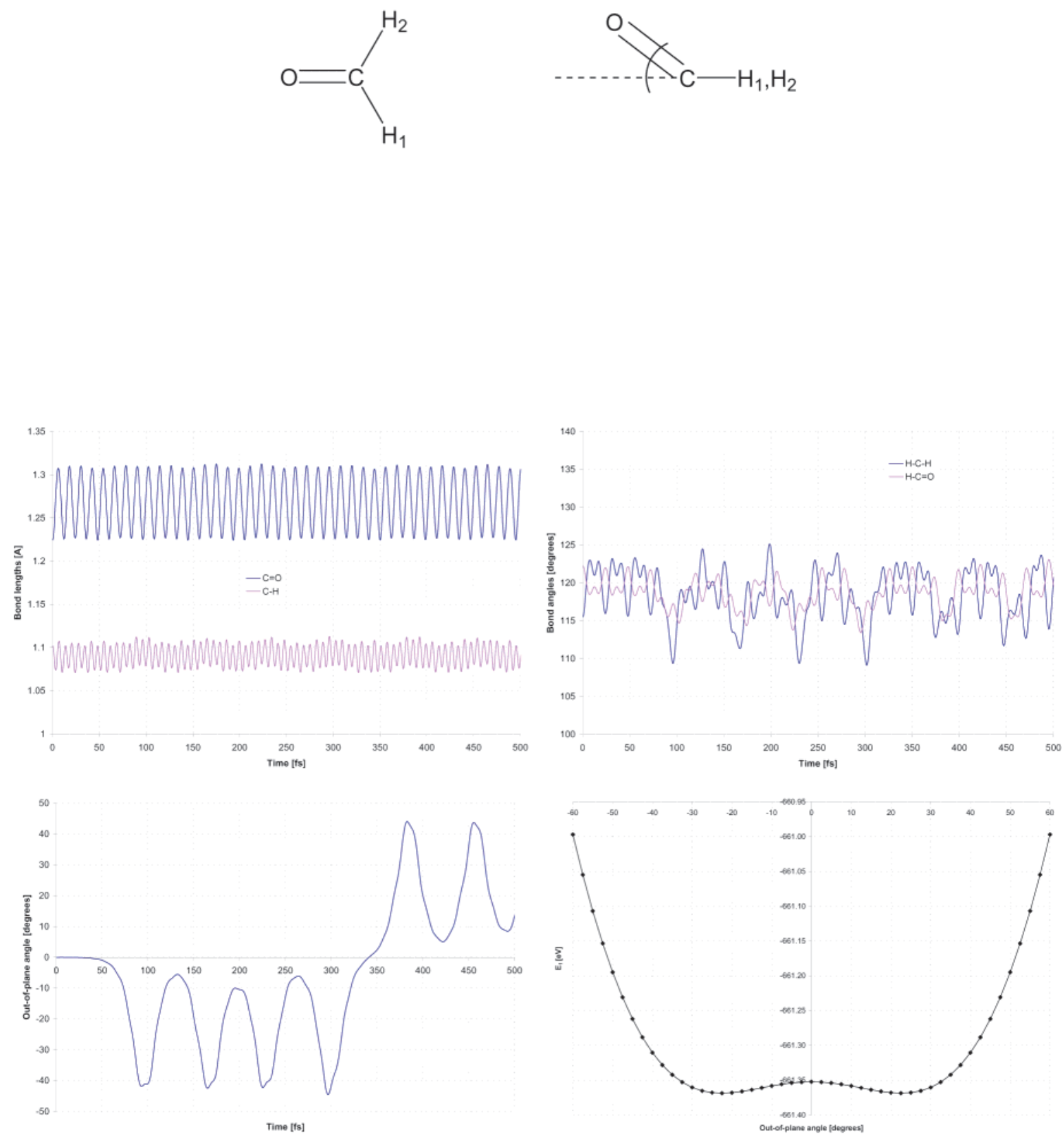

Figure 1. Upper panels: structure of the formaldehyde and definition of the out-of-plane angle. Middle panels: time evolution of the bond lengths and the bond angles following a vertical excitation from the ground-state equilibrium energy to the first excited state $(\Omega=3.83 \mathrm{eV})$. Lower panels: time evolution of the out-of-plane angle and potential energy curve of the first excited state along this angle.

We have tested the MDCEOV by performing molecular dynamics simulations on the first excited state of formaldehyde, which mainly involves the excitation of the electronic charge on the $\mathrm{C}=\mathrm{O}$ double bond. Analysis of the $\mathrm{CEO}$ eigenvector corresponding to the transition to this state shows that it corresponds to the HOMO-LUMO transition. We have computed a 500 fs trajectory following a vertical impulsive transition from the ground-state energy minimum $\left(C_{2 v}\right.$ point group $)$ to the first excited state. The results are shown in Figure 1. From the nature of the electronic excitation, we expect a major vibrational relaxation on the $\mathrm{C}=\mathrm{O}$ bond. The two $\mathrm{C}-\mathrm{H}$ bonds relax as well, but their vibrational amplitude is lower. Immediately following the excitation, we observe the shrinking of the $\mathrm{C}-\mathrm{H}$ bonds, together with the stretching of the $\mathrm{C}=\mathrm{O}$ bond. This can be rationalized by noting that excitation of the $\mathrm{C}=\mathrm{O}$ bond weakens this bond so that the excited state has a longer bond length. On the other hand, upon excitation, the electronic charge flux brings more bonding charge into the $\mathrm{C}-\mathrm{H}$ region, thus lowering the equilibrium $\mathrm{C}-\mathrm{H}$ distance in the excited state. At longer times, it is possible to follow the evolution of the out-of-plane angle formed by the $\mathrm{C}=\mathrm{O}$ bond with the plane defined by the two $\mathrm{C}-\mathrm{H}$ bonds. In the ground state, this angle is zero (planar geometry). The first excited state is non planar, but its gradient, in correspondence of the groundstate minimum geometry, does not have an out-of-plane component. The planar ground-state geometry thus corresponds to an unstable conformation of the first excited state. This can be inferred from the potential curve along the out-of-plane angle depicted in Figure 1. After $\sim 50$ fs, the out-of-plane bending vibration is activated by numerical noise, which finally brings some out-of-plane contributions to the gradient in the first excited state. In reality, the interaction of the excited molecule with the bath degrees of freedom should induce the out-of-plane motion. Note that the time-dependent out-of-plane coordinate shows an oscillation around an average angle of more than $20^{\circ}$, as expected from the potential minimum. 

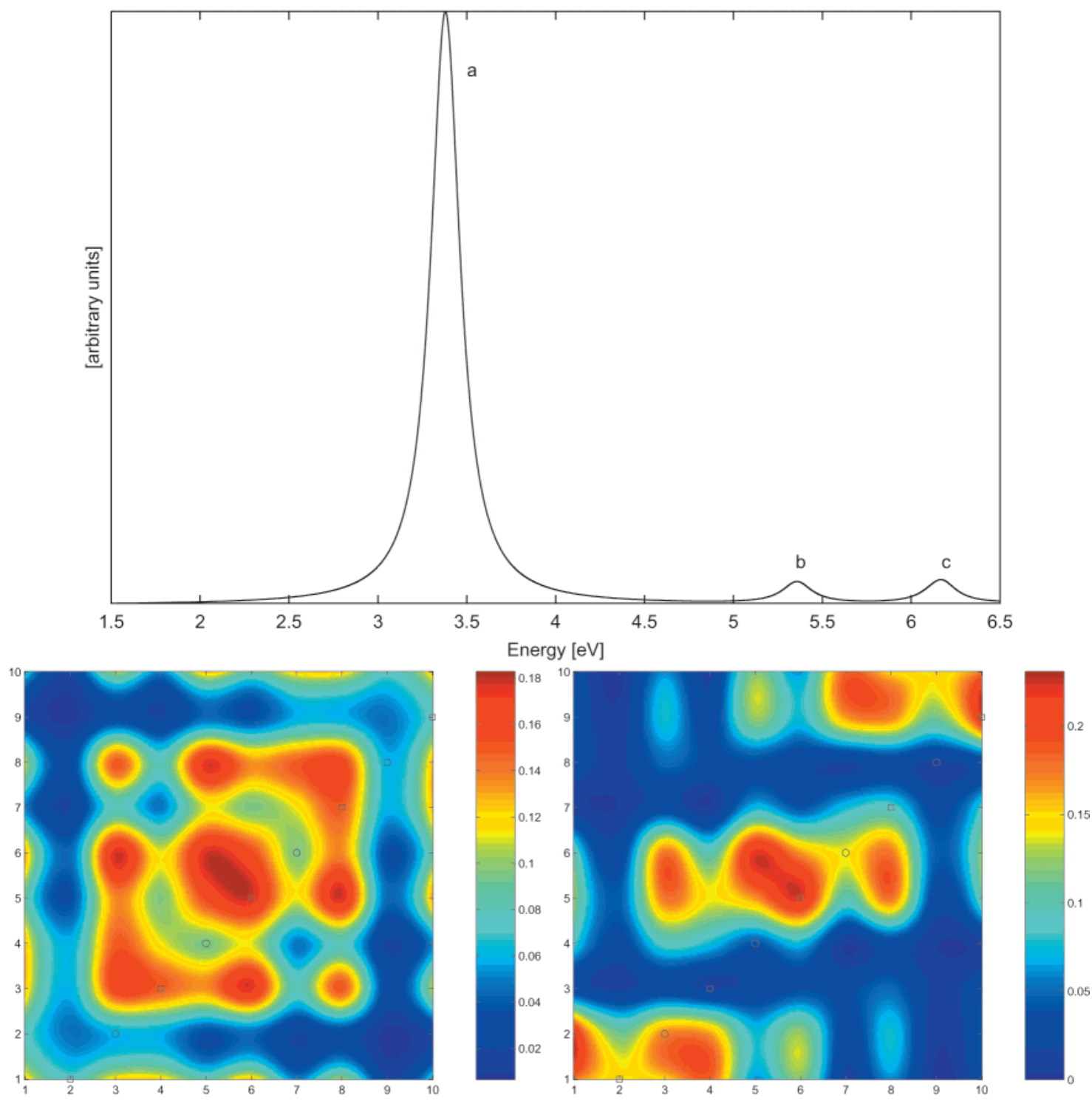

$$
\Omega_{a}=3.37 \mathrm{eV}
$$

$$
\Omega_{b}=5.35 \mathrm{eV}
$$

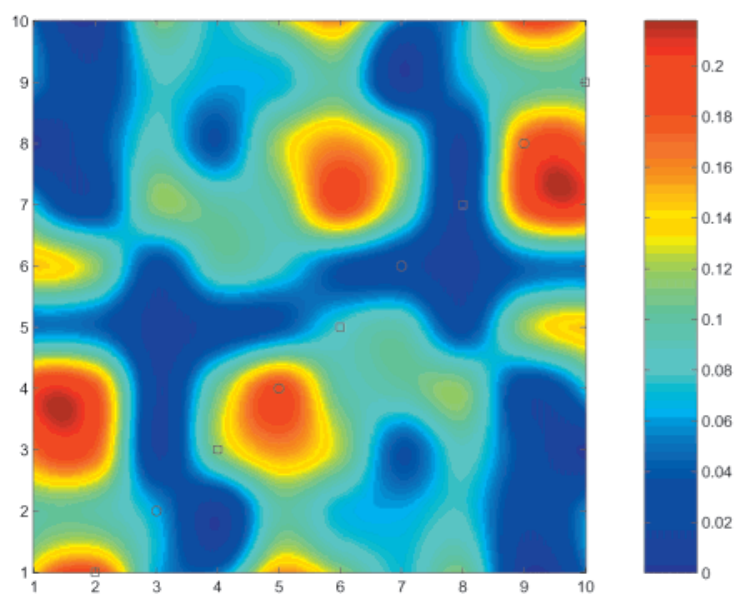

$$
\Omega_{c}=6.17 \mathrm{eV}
$$

Figure 2. Upper panel: normalized linear absorption spectrum of the polyacetylene oligomer with five double bonds. Lower panels: CEO plots of the electronic oscillators corresponding to the electronic transitions showing in the spectrum. See text for the description of the modes. 

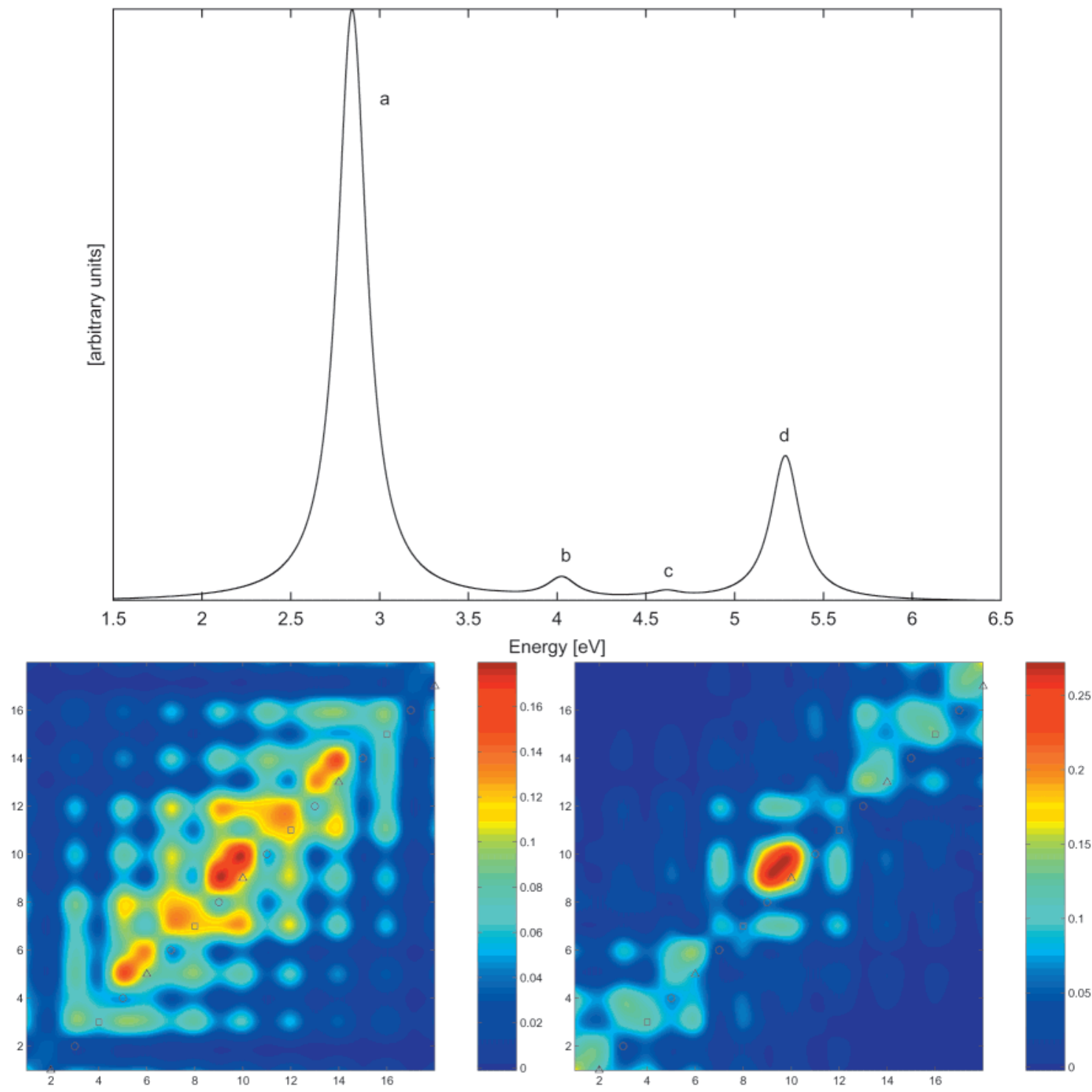

$$
\Omega_{a}=2.84 \mathrm{eV}
$$
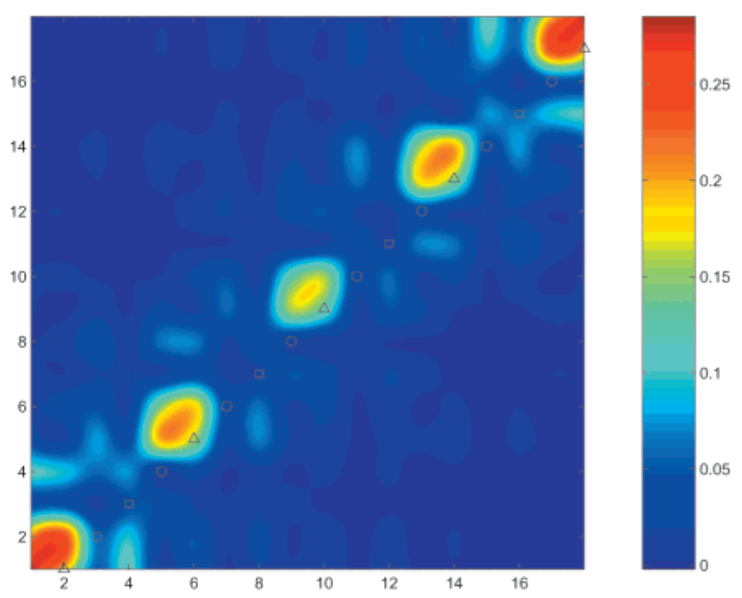

$$
\Omega_{b}=4.02 \mathrm{eV}
$$

$$
\Omega_{c}=4.61 \mathrm{eV}
$$

$\Omega_{d}=5.28 \mathrm{eV}$

Figure 3. Upper panel: normalized linear absorption spectrum of the polydiacetylene oligomer with five triple bonds. Lower panels: CEO plots of the electronic oscillators corresponding to the electronic transitions showing in the spectrum. See text for the description of the modes. 

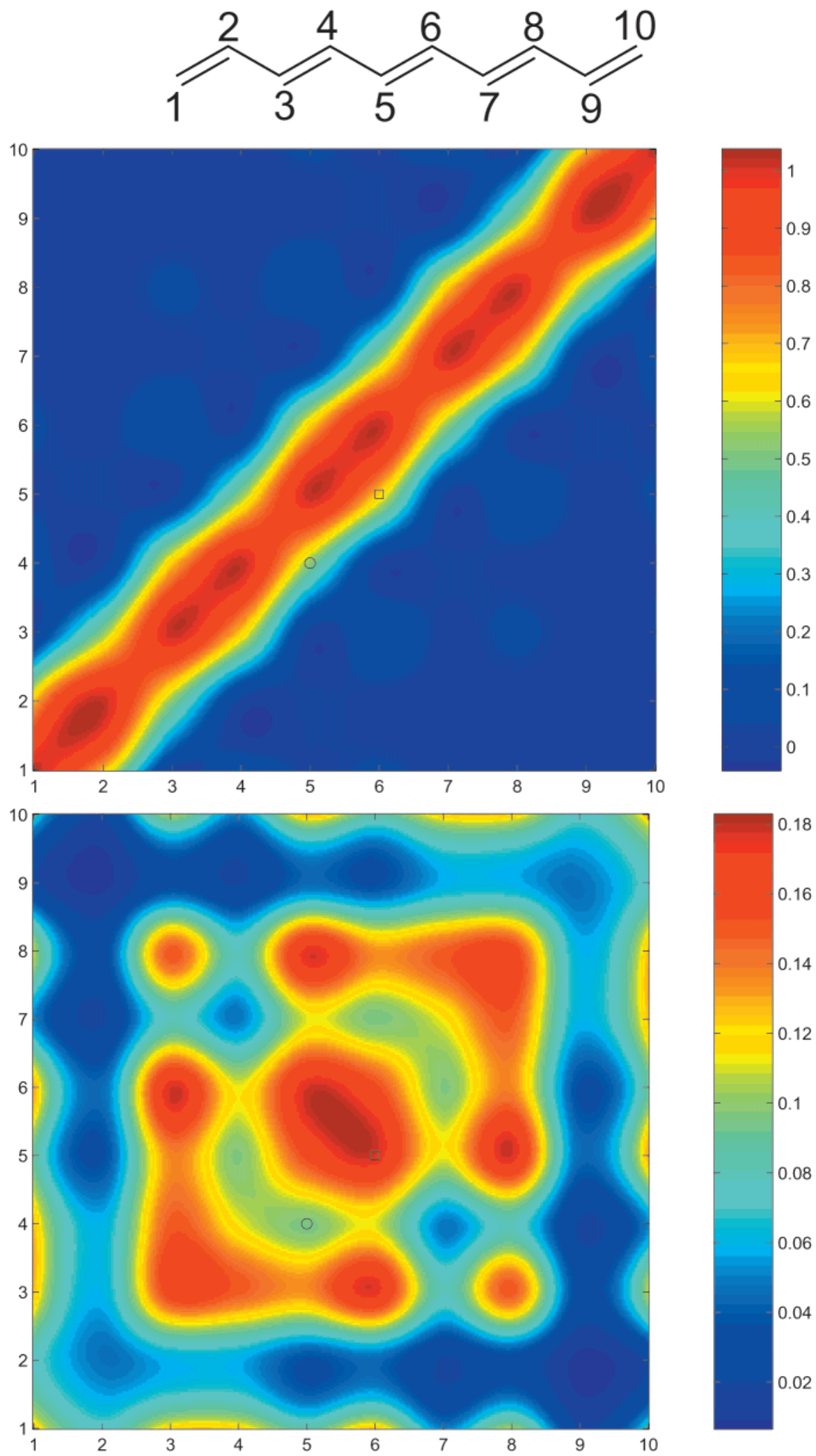

Figure 4. Upper panel: ground-state one-electron density matrix of the polyacetylene oligomer $\mathrm{C}_{10} \mathrm{H}_{12}$. Atom numbering is shown on the chemical structure. The circle and box markers point to the matrix elements corresponding to the central single and double bonds, respectively. Hydrogen atoms are not shown. Lower panel: one-electron transition density matrix for the lowest excited state $(\Omega=3.37 \mathrm{eV})$ of the polyacetylene oligomer.

\section{Application to Polyacetylene and Polydiacetylene Oligomers}

From the CEO calculation of the electronic excitations of a molecule, it is possible to obtain the linear response to an electric field, e.g. ,the UV-vis absorption spectra. ${ }^{22}$ This is shown in Figure 2 and Figure 3 for the two conjugated oligomers studied.
In analogy with vibrational spectroscopy, where the various transitions are related to nuclear normal modes, here each band can be related to an electronic oscillator which describes a collective electronic excitation. The modes corresponding to the dominant bands of the computed linear absorption spectra are also displayed in Figures 2 and 3. The plots of the CEO 
TABLE 1: Excitation Energies (eV) of the Lowest Excited State of Polyacetylene and Polydiacetylene Oligomers According to Several Computational Methods

\begin{tabular}{ccccc}
\hline & ZINDO/S-TDHF & INDO/S-CI & AM1/CAS-CI & ab initio CASPT2 ${ }^{32}$ \\
\hline PA oligomer & $3.37^{a}$ & $3.57^{b}$ & $3.86^{c}$ & - \\
PDA oligomer & $2.84^{a}$ & $3.03^{b}$ & - & $3.49^{d}$
\end{tabular}

${ }^{a}$ This work. ${ }^{b}$ Computed for the same oligomers studied in this work with the Argus code. ${ }^{c}$ Excitation energy for the PA oligomer with four double bonds. ${ }^{d}$ Excitation energy for the PDA oligomer with three triple bonds and four double bonds.

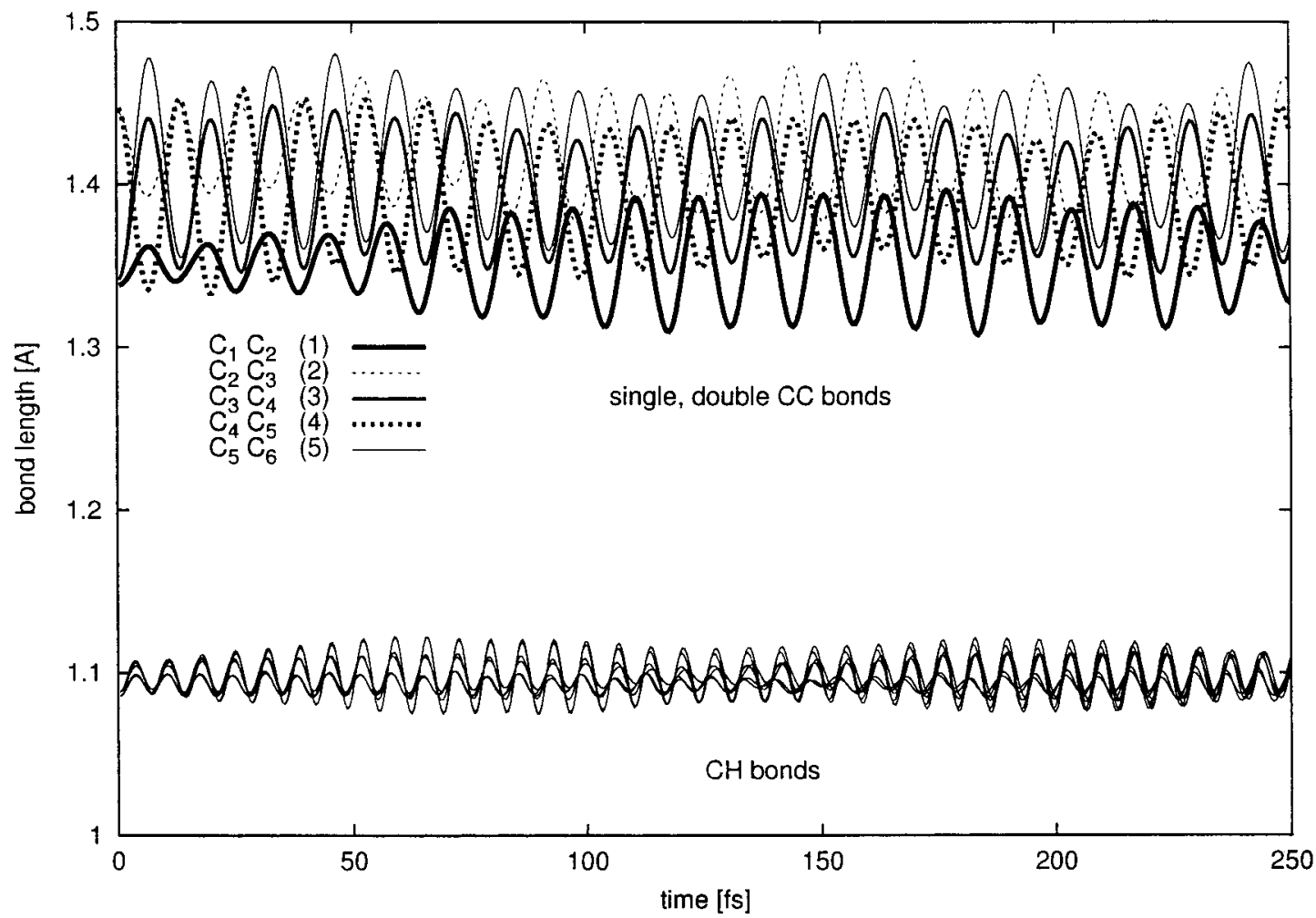

Figure 5. Bond length trajectories for the polyacetylene oligomer following an impulsive vertical excitation to the first allowed state $(\Omega=3.37$ $\mathrm{eV}$ ). Note the increased (reduced) lengths of double (single) bonds in the first few femtosecond, shown by the solid (dotted) lines. The amplitude of the bond length oscillations is determined by the difference in the equilibrium geometries between the excited state and the ground state. A time step of $0.5 \mathrm{fs}$ has been used in the MDCEOV computation.

eigenmodes are a suitable contraction of the eigenvector $\left(\xi_{v}\right)_{i j}$ corresponding to a given electronic transition characterized by the excitation energy $\Omega_{v}$. The contraction from the full atomic orbitals basis set $\left\{\chi_{i}\right\}$ to an atomic site representation $\{A\}$ is useful to ease the visualization of the nature of the excitation. The contraction scheme adopted here for a generic transition density matrix $\xi$ is

$$
\begin{gathered}
\xi_{A A}=\sum_{i \in A, j \in A} \xi_{i j} \\
\xi_{A B}=\left[\sum_{i \in A, j \in B} \xi_{i j}^{2}\right]^{1 / 2} A \neq B
\end{gathered}
$$

The diagonal matrix elements in the atomic site representation, $\xi_{A A}$, are given by the sum of the elements of $\xi$ pertaining to the atomic orbitals centered on the atom $A$, whereas the off diagonal elements, $\xi_{A B}$, are related to the sum of the squares of the matrix elements of $\xi$ corresponding to the atomic orbitals centered on $A$ and on $B$, respectively. The diagonal elements of $\xi$ represent the atomic charges induced upon excitation, and the off-diagonal elements are known as coherences. The coherence between two chemically bonded atoms is related to the variation of the bond order induced by the electronic excitation. The same contraction scheme may be also used to represent the ground-state density matrix, $\bar{\rho}$. This representation of the electronic oscillators has been used in previous CEO studies of optical excitations of conjugated systems. ${ }^{21-23}$ Figures $2-4$ and 8 display the color maps of the contracted elements of the CEO transition density matrices contracted in the atomic site representation. The hydrogen atoms are not shown in the plot since the excitations studied involve mainly the $\pi$ electrons on the carbon atoms. A cubic interpolation algorithm has been applied to smooth the color changes in the plot. Off-diagonal elements corresponding to coherences among bonded atoms are marked according to the order of the bond: single $(\bigcirc)$, double $(\square)$, or triple $(\triangle)$. Coherences among nonbonded atoms can be observed in the plots as well, as for example between atoms 3 and 8 in Figure 4. The presence (absence) of coherences between distant nonbonded atoms indicates the degree of localization of the excited state.

In Table 1, we compare the lowest excitation energies obtained using the CEO and other methods. The agreement is good, in particular with respect to the Argus results. ${ }^{31}$ The results from the other computational methods are reported as a reference, since the molecules studied in refs 32 and 33 slightly differ from the ones analyzed in the present work (they have a shorter conjugation length, thus leading to higher excitation energies).

The absorption spectrum of the trans-polyacetylene oligomer with five double $\mathrm{C}=\mathrm{C}$ bonds $\mathrm{C}_{10} \mathrm{H}_{12}$ computed using the $\mathrm{CEO}$ 


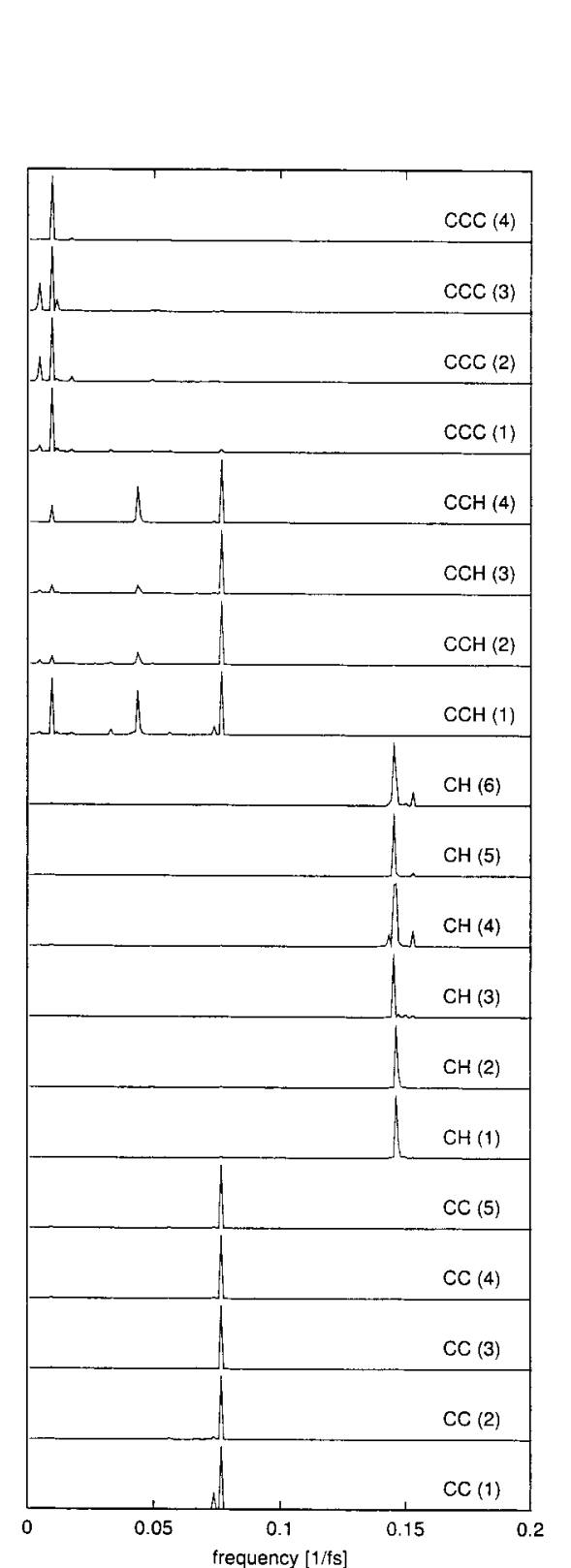<smiles>CC(C)=C(C)C(C)=C(C)C(C)=C(C)C(C)=C(C)C</smiles><smiles>CC(C)=C(C)C(C)=C(C)C(C)=C(C)C(C)=C(C)C</smiles><smiles>CC(C)=C(C)C(C)=C(C)C(C)=C(C)C(C)=C(C)C(C)C</smiles><smiles>CC(C)=C(C)C(C)=C(C)C(C)=C(C)C(C)=C(C)C</smiles>

Figure 6. Fourier transform of the trajectory of Figure 5 and of the time evolution of the relevant bond angles; notice that the spectra show no strong coupling of the stretching degrees of freedom with lower-frequency bending motions (CCC bendings).

approach is shown in Figure 2; the spectrum has three peaks. Peak a corresponds to the HOMO-LUMO transition, and it has a strong intensity. The associated CEO mode represents a collective excitation of the central single and double bonds ranging from atom 3 to atom 8 . This mode has a coherence size (off-diagonal spread of the mode) of about $6 \AA$, corresponding to the distance between atoms 3 and 8 . Peak $b$ represents a more independent excitation of the central double bond and the two terminal double bonds. A strong coherence is present between atom 3 and atoms 5 and 6 , forming the central double bond; in a symmetric way, coherence is observed also between atom 8 and the atoms of the central double bond. Finally, peak $\mathrm{c}$ is the excitation on the central single bonds (e.g., the 4,5 and 6,7 bonds) with an associated coherence involving the atom 1,2 and the atoms 3 and 4 (and symmetrically atoms 9,10 and 7,8 )

The absorption spectrum of the PDA oligomer shown in Figure 3, together with the CEO eigenvectors. The spectrum shows four transitions. The a peak is a strong band corresponding to the HOMO-LUMO transition. The associated CEO mode shows an exitation spreading over the three central triple bonds, and it also involves the two central double bonds, as can be inferred by the triangle and square markers which correspond to the position of the triple and double bonds, respectively. This excitation has a maximum coherence size of $6 \AA$, corresponding to the distance between the carbon atoms 12 and 7. The coherence size is determined by the extent of the interactions among $\pi$ electrons and in our case is ultimately determined by the matrix elements of the ZINDO/S Hamiltonian ( $t$ and $V$ ). In the case of the a mode, the exciton size is smaller than the molecular physical size; the excitation thus resides mainly on the center of the oligomer and does not extend appreciably to the ends. The higher-energy modes of the PDA oligomer are more localized. They consist of various combinations of excitations centered on the triple bonds. In particular, mode $b$ is mainly the excitation of the central triple bond, whereas mode 


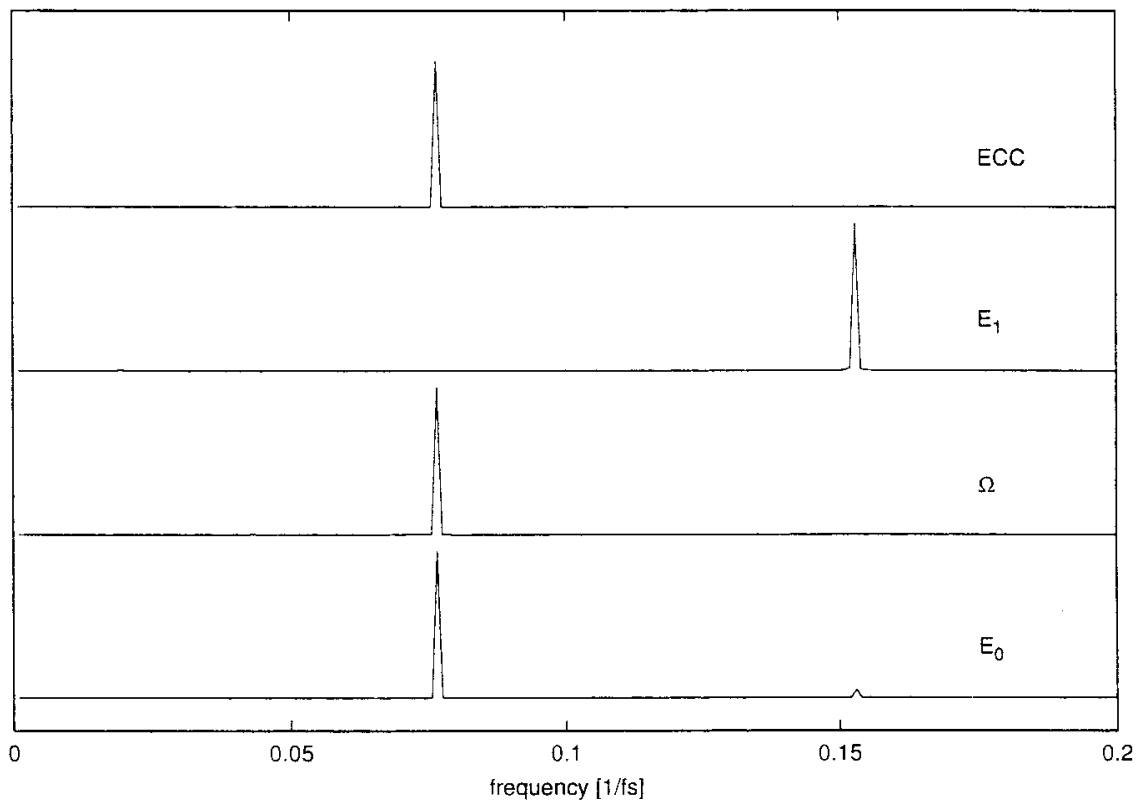

Figure 7. Fourier transform of the trajectories of the ground-state energy $E_{0}$, the excitation energy $\Omega$, the first excited-state energy $E_{1}$, and the R coordinate for the polyacetylene oligomer (see text for details).

c represents the excitation of the terminal triple bonds. Finally, mode $d$ is the collective excitation of the three central triple bonds, with a stronger excitation on the central bond.

We have performed molecular dynamics computations on the PA oligomer, which is known to be long enough to show the features of the ECC coordinate. ${ }^{16} \mathrm{CEO}$ computations show that the first excited state of this system has a large contribution from the HOMO-LUMO transition. In polyenes, the HOMO charge distribution is centered on the double $\mathrm{C}=\mathrm{C}$ bonds, whereas the LUMO is centered on the single $\mathrm{C}-\mathrm{C}$ bonds. Upon excitation, we thus expect some bond charge to move from the double bonds to the single bonds. This implies that the single $\mathrm{C}-\mathrm{C}$ bonds in the excited state are stronger than those in the ground state, whereas the double $\mathrm{C}=\mathrm{C}$ bonds are weaker. For that reason, the equilibrium double-bond (single bond) length is longer (shorter) in the excited state. We thus expect that upon excitation the main nuclear relaxation process should involve the stretching of carbon-carbon bonds. This is supported by the CEO transition density matrix for the first excited state displayed in Figure 4. The markers on the CEO plots show the matrix elements corresponding to the bond order change for double and single bonds. The figure shows that upon excitation the bond order changes both for single and double bonds. This is accompanied by an induced change of the atomic charges upon excitation, as shown by the diagonal elements. The spread of the excitation in the molecule can be estimated by looking at the distribution of the large elements in the transition density matrix along the diagonal and the antidiagonal directions. For this polyacetylene oligomer, the excitation extends mainly from atom 3 to atom 8 , covering a coherence length of 3 double bonds.

We have studied the nuclear relaxation following an impulsive excitation from the ground state to the first allowed excited state, with a transition energy of $3.37 \mathrm{eV}$. The time evolution of the $\mathrm{CC}$ and $\mathrm{CH}$ bond lengths is shown in Figure 5. A Fourier transform of the carbon-carbon and carbon-hydrogen bond length trajectories depicted in Figure 6 shows no strong interaction between carbon-carbon stretching and CCC bending; the Fourier spectra of the CC stretching do not show any other peak except that for the CC vibration. On the other hand, the spectra of the $\mathrm{CCH}$ bendings contain a strong $\mathrm{CC}$ stretching component. This behavior is different from that of polydiacetylene, where carbon-carbon stretchings and $\mathrm{CCC}$ bendings along the chain are coupled, as will be illustrated below. Our computations further show that the ultrafast nuclear relaxation following an ideal impulsive excitation is dominated by the ECC coordinate: the behavior of the energy along the trajectory can be explained using this $\mathrm{R}$ relaxation coordinate. To that end, we introduce a simple harmonic model for the excited-state dynamics

$$
\begin{gathered}
E_{0}=\frac{1}{2} k \mathrm{R}^{2} \quad E_{1}=\Omega_{0}+\frac{1}{2} k\left(\mathrm{R}-\mathrm{R}_{1}\right)^{2} \\
\Omega=E_{1}-E_{0}=\Omega_{0}+\frac{1}{2} k \mathrm{R}_{1}^{2}\left(1-2 \frac{\mathrm{R}}{\mathrm{R}_{1}}\right)
\end{gathered}
$$

We set the zero of the energy scale and of $R$ at the groundstate minimum. $R$ is the relevant vibrational coordinate which couples the ground and the first excited state, and $k$ is its force constant. It is defined through a linear combination of $\mathrm{C}-\mathrm{C}$ $\left(R_{i}\right)$ and $\mathrm{C}=\mathrm{C}\left(r_{i}\right)$ stretchings, which are out of phase

$$
\begin{aligned}
& \mathrm{R}= \\
& \frac{\alpha_{1} r_{1}-\alpha_{2} R_{2}+\alpha_{3} r_{3}-\alpha_{4} R_{4}+\alpha_{5} r_{5}-\alpha_{6} R_{6}+\alpha_{7} r_{7}-\alpha_{8} R_{8}+\alpha_{9} r_{9}}{\sqrt{\alpha_{1}^{2}+\alpha_{2}^{2}+\alpha_{3}^{2}+\alpha_{4}^{2}+\alpha_{5}^{2}+\alpha_{6}^{2}+\alpha_{7}^{2}+\alpha_{8}^{2}+\alpha_{9}^{2}}}
\end{aligned}
$$

The coefficients $\alpha_{i}$ can be computed using the output from the molecular dynamics computation. Let us denote by $A\left(L_{i}\right)$ the mean amplitude of oscillation of a given bond length $L_{i}$. If the bond length dynamics is harmonic, the amplitude is given by

$$
A\left(L_{i}\right)=\sqrt{2} \sqrt{\frac{\int_{0}^{T}\left[L_{i}(t)-\left\langle L_{i}\right\rangle\right]^{2} \mathrm{~d} t}{T}}
$$

Here $\left\langle L_{i}\right\rangle$ is the time-averaged bond length during the time interval $T$ 

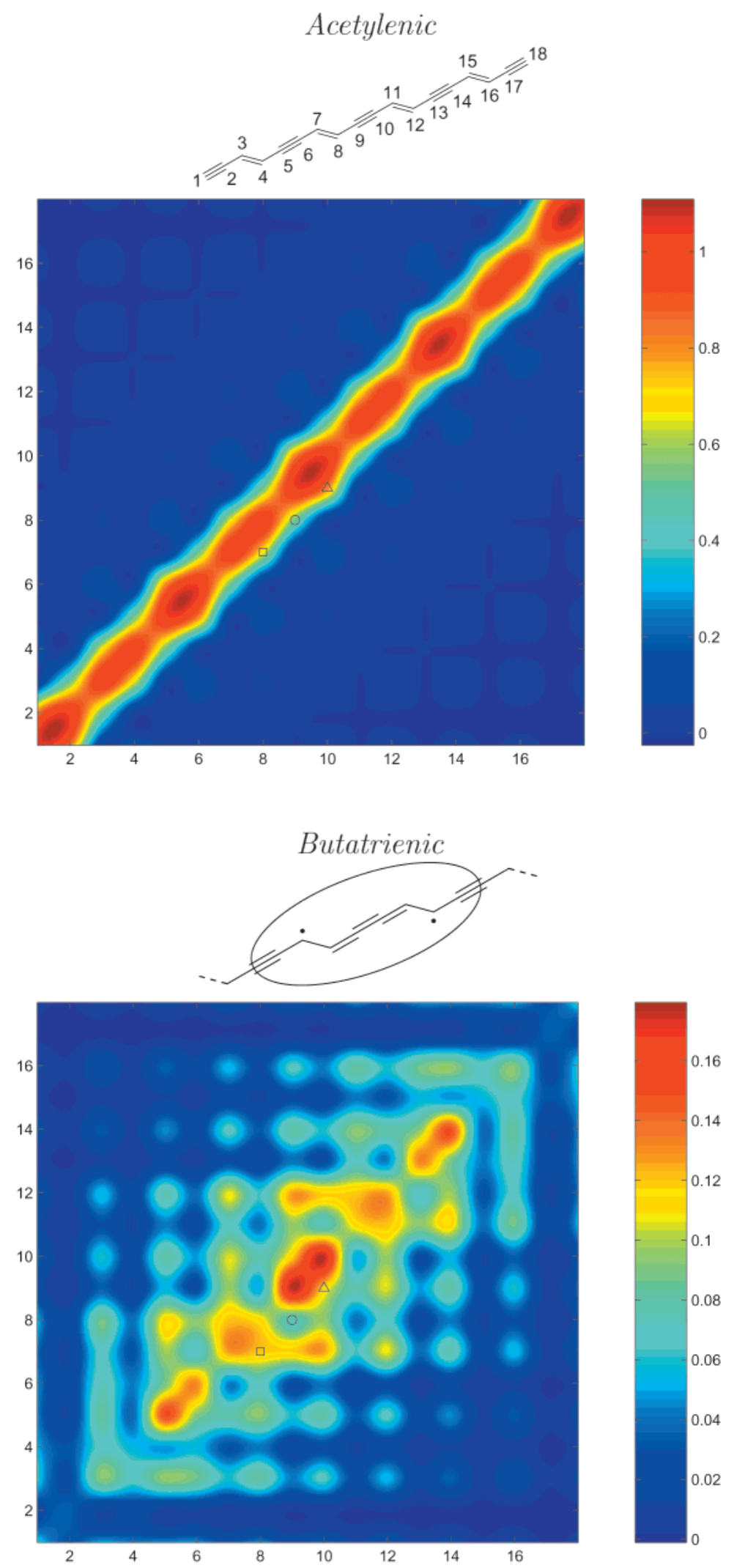

Figure 8. Upper panel: single electron density matrix for the ground state of the PDA oligomer. The circle, box, and triangle markers point to the matrix elements corresponding to the central single, double, and triple bonds, respectively. Hydrogen atoms are not shown. Lower panel: single electron transition density matrix for the lowest electronic excitation of the PDA oligomer at $\Omega=2.8 \mathrm{eV}$.

$$
\left\langle L_{i}\right\rangle=\frac{1}{T} \int_{0}^{T} L_{i}(t) \mathrm{d} t
$$

Using a set of bond lengths $\left\{L_{i}\right\}$ that enter the definition of an ECC coordinate for a given molecule, we can express the

coefficients $\alpha_{i}$ as

$$
\alpha_{i}=\frac{A\left(L_{i}\right)}{A_{\max }}
$$




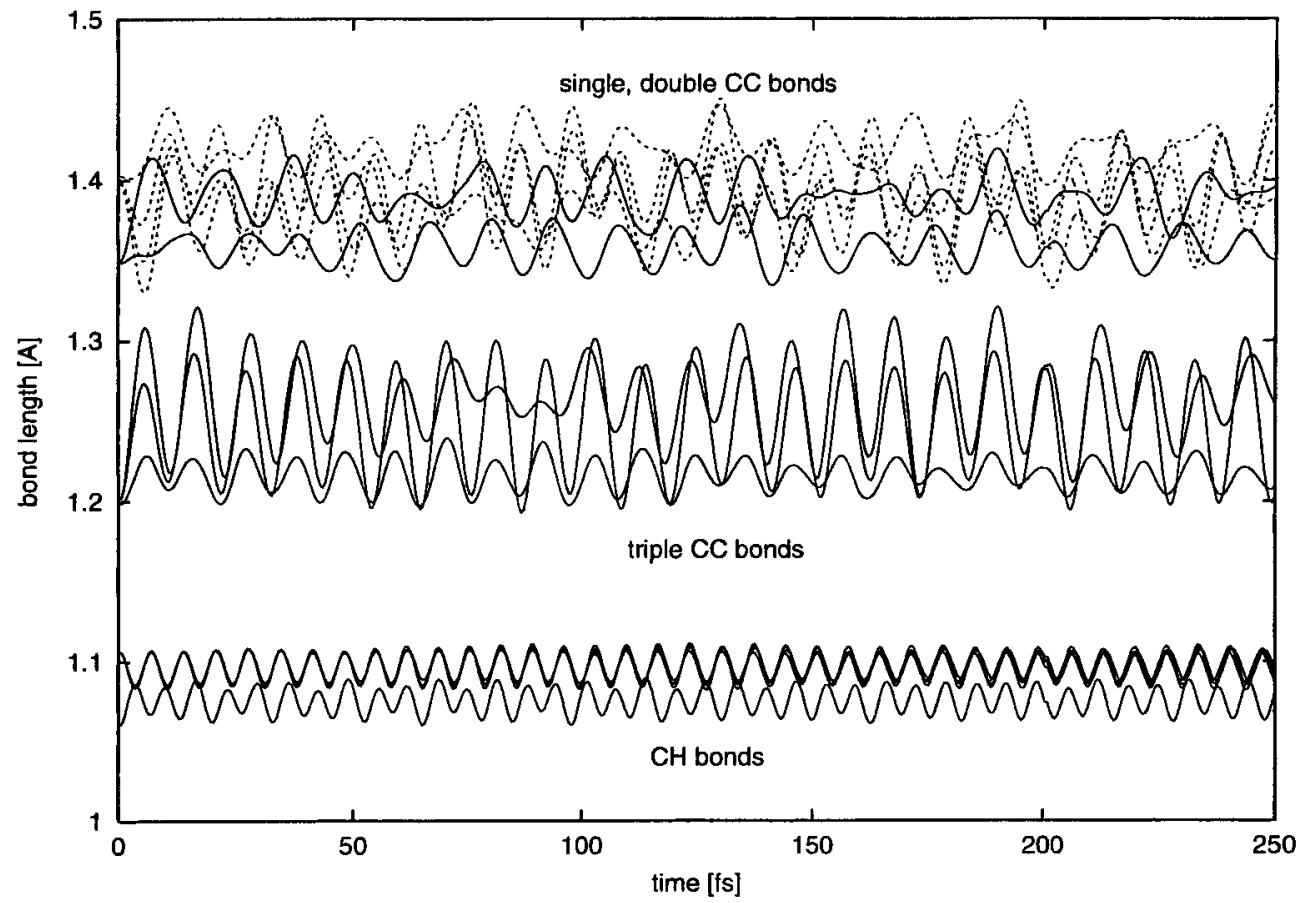

Figure 9. Time dependence of the various bond lengths using the excited-state trajectories of the PDA oligomer. In the upper trace, single (double) carbon-carbon bonds are represented by the dotted (solid) lines. For this impulsive excitation from the ground state, the amplitude of the bond length oscillation is determined by the difference in the equilibrium geometries between the excited state and the ground state. A time step of 0.5 fs has been used in the MDCEOV computation.

TABLE 2: Coefficients $\alpha_{i}$ for the Carbon-Carbon Stretching Coordinates in the Definition of the ECC Obtained from the Molecular Dynamics Simulations ${ }^{a}$

\begin{tabular}{cccccc}
\hline bond & $\mathrm{C}_{1}=C_{2}$ & $\mathrm{C}_{2}-\mathrm{C}_{3}$ & $\mathrm{C}_{3}=\mathrm{C}_{4}$ & $\mathrm{C}_{4}-\mathrm{C}_{5}$ & $\mathrm{C}_{5}=\mathrm{C}_{6}$ \\
\hline$\alpha_{i}, i=1-5$ & 0.62 & 0.73 & 0.90 & 0.98 & 1.00
\end{tabular}

${ }^{a}$ See text and eq 24 . The coefficients $\alpha_{i}(i=6-9)$ are obtained by symmetry, i.e., $\alpha_{6}=\alpha_{4}, \alpha_{7}=\alpha_{3}, \alpha_{8}=\alpha_{2}$, and $\alpha_{9}=\alpha_{1}$. The numbering of the atoms is shown in Figure 4.

$A_{\max }$ is the maximum amplitude of vibration in the given set of bonds. The coefficients $\alpha_{i}$ for $\mathrm{C}_{10} \mathrm{H}_{12}$ obtained from the carboncarbon bond lengths trajectories are given in Table 2. The solution of the equation of motion for the ECC

$$
m \ddot{\mathrm{R}}=-\frac{\mathrm{d} E_{1}}{\mathrm{~d} t}=-k\left(\mathrm{R}-\mathrm{R}_{1}\right)
$$

with the initial conditions $R(0)=0$ and $R^{\dot{R}}(0)=0$ are

$$
\mathrm{R}(t)=\mathrm{R}_{1}[1-\cos (\omega t)]
$$

where $\omega^{2}=\mathrm{k} / \mathrm{m}$. Substituting $\mathrm{R}(t)$ into eq 23, we obtain the time evolution of the ground-state, excited-state, and excitation energies

$$
\begin{gathered}
E_{0}(t)=\frac{1}{4} k \mathrm{R}{ }_{1}^{2}[3-4 \cos (\omega t)+\cos (2 \omega t)] \\
E_{1}(t)=\Omega_{0}+\frac{1}{4} k \mathrm{R}{ }_{1}^{2}[1+\cos (2 \omega t)] \\
\Omega(t)=\Omega_{0}+\frac{1}{4} k \mathrm{R}{ }_{1}^{2}[4 \cos (\omega t)-2]
\end{gathered}
$$

A Fourier transform performed on these trajectory for these quantities of interest $\left(E_{0}, E_{1}, \Omega\right.$, and $\left.\mathrm{R}\right)$ is shown in Figure 7 . The transition energy $\Omega$ oscillates with the same frequency $(\omega)$ of $\mathrm{R}$, whereas the excited-state energy $E_{1}$ oscillates at twice this frequency. The ground-state energy $E_{0}$ has a strong $(\omega)$ and a much weaker $(2 \omega)$ component. These observations confirm the simple picture offered by the model of eq 30 .

Pump-probe experiments performed recently by Kobayashi and co-workers on polydiacetylene (PDA) ${ }^{24}$ revealed details of the geometry relaxation on the excited state. This includes the geometry change from acetylenic to butatrienic geometry involving carbon-carbon stretchings strongly coupled with inplane bendings along the chain. This change has been recently studied by ab initio computations. ${ }^{32}$ We applied MDCEOV to explore the dynamics of the excited state of a PDA oligomer. By simulating an ideal vertical excitation from the equilibrium ground-state geometry to the lowest excited state, characterized by a vertical excitation energy $\Omega=2.8 \mathrm{eV}$, we followed the classical nuclear dynamics on this excited-state surface for 1 ps. The first few femtosecond of the evolution of the carboncarbon bonds in the center of the oligomer show lengthening of triple bonds and shortening of single bonds. This is a consequence of the butatrienic form of the excited state, where the single and the triple bonds become double bonds with shorter and longer new bond equilibrium geometries, respectively (see Figure 8). We examined the time evolution of the molecular geometry by following the bond lengths and angles. The bond lengths are depicted in Figure 9. The $\mathrm{CH}$ stretching vibrations behave as simple uncoupled harmonic oscillators. This is to be expected since high-frequency vibrations are decoupled from other vibrational degrees of freedom. The time evolution of carbon-carbon bond lengths is more involved since they are more strongly coupled among themselves and with the bendings. To analyze the nature of the dynamics of the carbon-carbon stretchings, we showed in Figure 10 a Fourier analysis of the bond lengths and bond angles in the center of the molecule, where the changes induced by the electronic excitation are the largest. The $\mathrm{CH}$ stretching vibrations appear isolated in the spectrum, as suggested by the trajectory. In contrast, strong interactions are clearly seen between the $\mathrm{CC}(2)$ stretch and the 

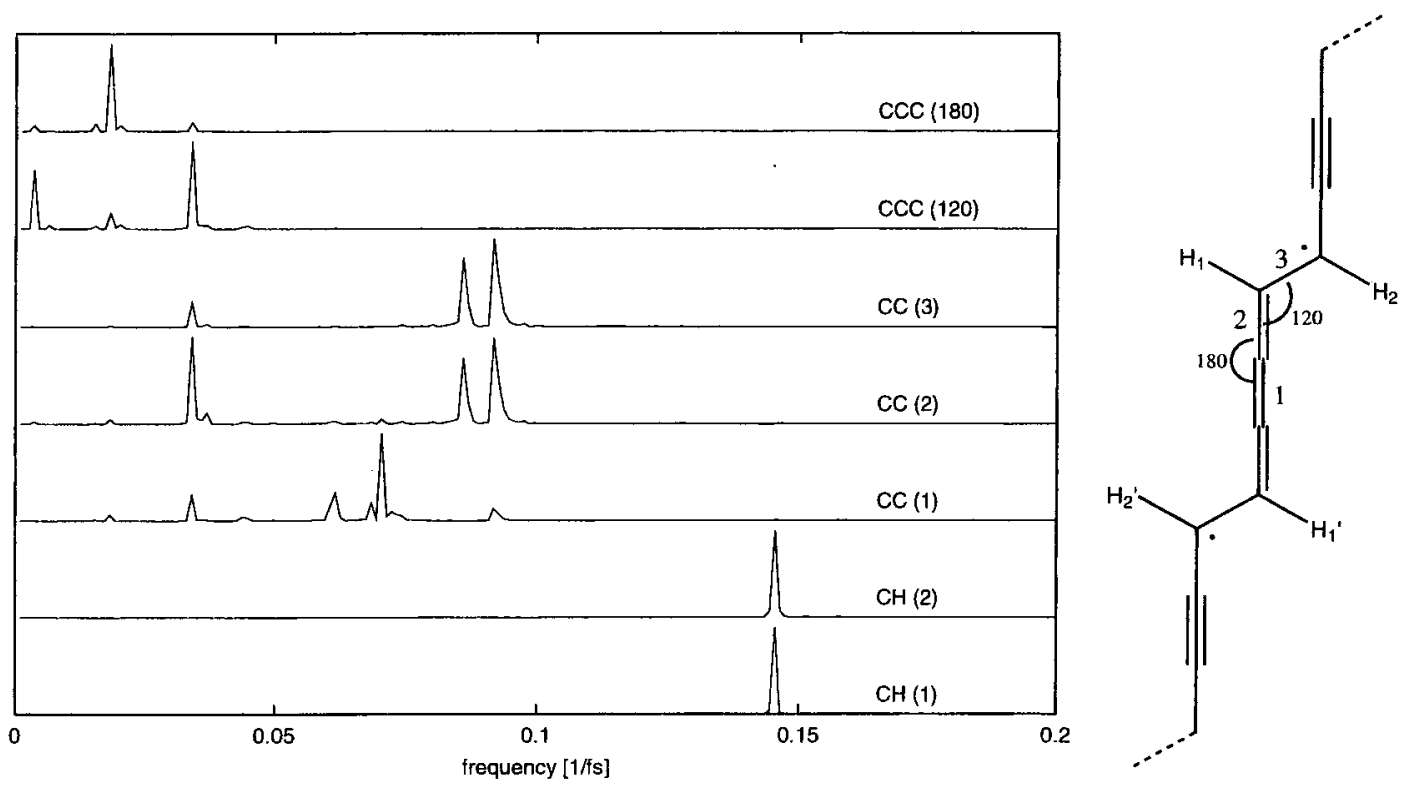

Figure 10. Fourier transform of the time evolution of several bond lengths and bond angles at the center of the PDA oligomer. The labels of the internal coordinates considered are shown in the right.

$\mathrm{CCC}(120)$ bending: a strong peak at the main bending vibration frequency appears in the spectrum of the $\mathrm{CC}(2)$ stretching. Weaker interactions can also be observed between $\mathrm{CC}(1)$, $\mathrm{CC}(3)$ vibrations, and the $\mathrm{CCC}(120)$ bending. $\mathrm{CC}(2)$ and $\mathrm{CC}(1)$ vibrations are also weakly coupled: a peak with the main $\mathrm{CC}(2)$ vibration frequency appears in the spectrum of the $\mathrm{CC}(1)$ vibration. These results, in particular the stretchingbending coupling in the dynamics of the excited state, compare favorably with experiment. ${ }^{24}$

To further analyze the dynamics, we carried out a vibrational normal-mode analysis at the minimum of the excited-state surface. The required second derivatives of the energy with respect to the nuclear coordinates have been computed numerically from the knowledge of the gradient. The position of the minimum has been estimated as the time average of the coordinates during the dynamics, i.e., $\mathbf{x}_{0} \approx\langle\mathbf{x}(t)\rangle$. This is strictly true for the dynamics of a displaced harmonic oscillator, but we expect it to be a good approximation more generally. We checked the computed Hessian and found only a few negative eigenvalues of small magnitude, indicating that this is a good estimate for the energy minimum. The vibrational normal coordinates $q_{i}(t)$ can then be used to express the nuclear dynamics

$$
\mathbf{x}(t)=\mathbf{x}_{0}+\sum_{i} q_{i}(t) \mathbf{L}_{i}
$$

$\mathbf{L}_{i}$ is a vector in the nuclear coordinates space representing the direction of motion in correspondence of the $i$ th normal mode. The vectors $\mathbf{L}_{i}$ can be easily computed from the knowledge of the Hessian matrix, as discussed in ref 34 . The projection of the dynamics $\mathbf{x}(t)$ on three Raman active normal modes $\mathrm{a}, \mathrm{b}$, and $\mathrm{c}$ is shown in Figure 11. The normal coordinates shown are normalized with respect to the amplitude $A_{c}$ of the mode c, i.e., we plot $q_{\mathrm{a}}(t) / \mathrm{A}_{\mathrm{c}}, q_{\mathrm{b}}(t) / \mathrm{A}_{\mathrm{c}}$, and $q_{\mathrm{c}}(t) / \mathrm{A}$ c. The vectors $\mathbf{L}_{\mathrm{a}}$, $\mathbf{L}_{b}$, and $\mathbf{L}_{\mathrm{c}}$ are also shown in Figure 11. Mode a is the in-phase $\mathrm{C} \equiv \mathrm{C}$ stretching, whereas mode $\mathrm{b}$ is the out-of-phase stretching of the central $\mathrm{C} \equiv \mathrm{C}$ bond with respect to all other triple bonds. Mode $\mathrm{c}$ is a collective angular motion involving the backbone of the molecule and corresponds to a contraction/elongation along the chain axis. It is reminiscent of the LAM (Longitudinal Acoustic Mode) motion, well-known in the vibrational dynamics of polymers. ${ }^{35}$ The normalized coordinates $q(t) / \mathrm{A}_{\mathrm{c}}$ of Figure 11 show the coupling between $\mathrm{C} \equiv \mathrm{C}$ stretching motions and angular motions (i.e., mode c). This behavior is consistent with the previous analysis, in particular with the stretching-bending coupling shown in the Fourier transform of the internal coordinates (Figure 10).

In conclusion, we have demonstrated how a molecular dynamics simulation combined with the electronic density matrix can be effectively used for computing and analyzing the nature of photoexcitations in conjugated oligomers. A notable advantage of the CEO approach is that information about the entire manifold of excited states can be easily generated in parallel at each step. The evolution of the system can then be computed in parallel on several excited-state surfaces. Nonadiabatic couplings $A_{v 0}^{x_{\mathrm{a}}}=-\left(\xi_{v} \mid \partial \bar{\rho} / \partial x_{\alpha}\right)$ may be readily computed in the TDHF/CEO framework. ${ }^{36,37}$ This provides a considerable computational advantage since these couplings are essential for simulating processes occurring near conical intersections, such as photoisomerization reactions.

Acknowledgment. This work was supported by the Chemical Sciences Division of the Office of Basic Energy Sciences of the DOE. Funding from the Italian Ministry for Scientific Research (MURST) is also acknowledged. We wish to thank Dr. C. Castiglioni for many fruitful discussions.

\section{Appendix A: The excitation energy gradient}

A closed expression for the derivative of the excitation energy $\Omega_{v}$ can be obtained by differentiating the CEO eigenvalue equation, taking into account the derivative of the normalization condition, $\left(\xi_{v} \mid \xi_{v}\right)=1$, of the electronic normal modes. It follows from the definition of the scalar product between Liouville space vectors $\mid \xi), \mid \eta)(\xi \mid \eta)=\operatorname{Tr}\left(\bar{\rho}\left[\xi^{\dagger}, \eta\right]\right)$ that $\left(\xi_{v}^{x} \mid \xi_{v}\right)+\left(\xi_{v} \mid \xi_{v}^{x}\right)+$ $\operatorname{Tr}\left(\bar{\rho}^{x}\left[\xi_{v}^{\dagger}, \xi_{v}\right]\right)=0$. Since the matrix $\bar{\rho}^{x}\left[\xi_{v}^{\dagger}, \xi_{v}\right]$ belongs to the particle-hole subspace, it is traceless, and we have $\left(\xi_{v}^{x} \mid \xi_{v}\right)+$ $\left(\xi_{v} \mid \xi_{v}^{x}\right)=0$. [It can be shown that $\bar{\rho}^{x}$ is a particle-hole (interband) matrix. ${ }^{19}$ The commutator of two interband matrices $\xi_{v}^{\dagger}$ and $\xi_{v}$ is an intraband matrix. Finally the product of an interband matrix and an intraband matrix is a traceless interband matrix.] The excitation energy is given by the following 


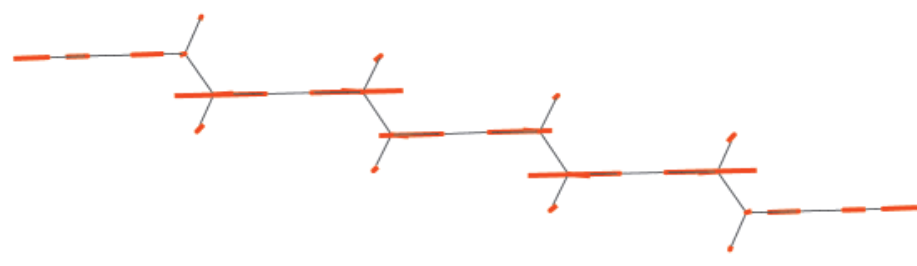

(a)

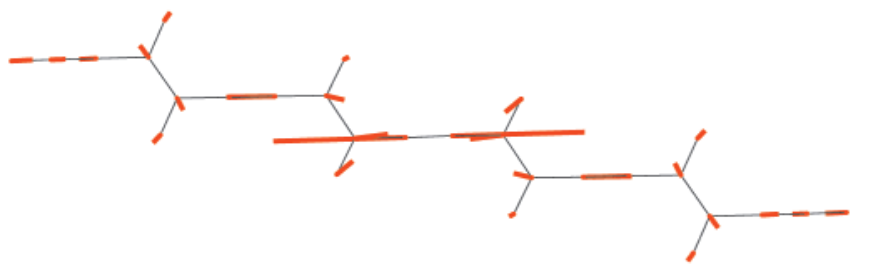

(b)

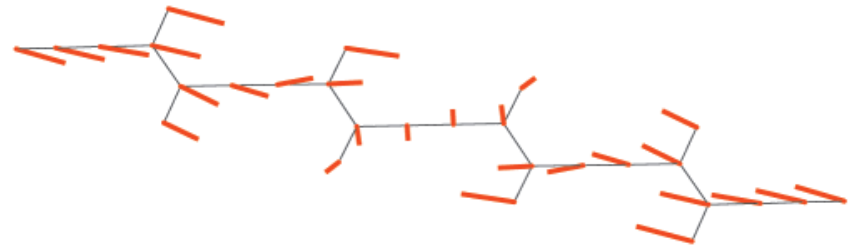

(c)

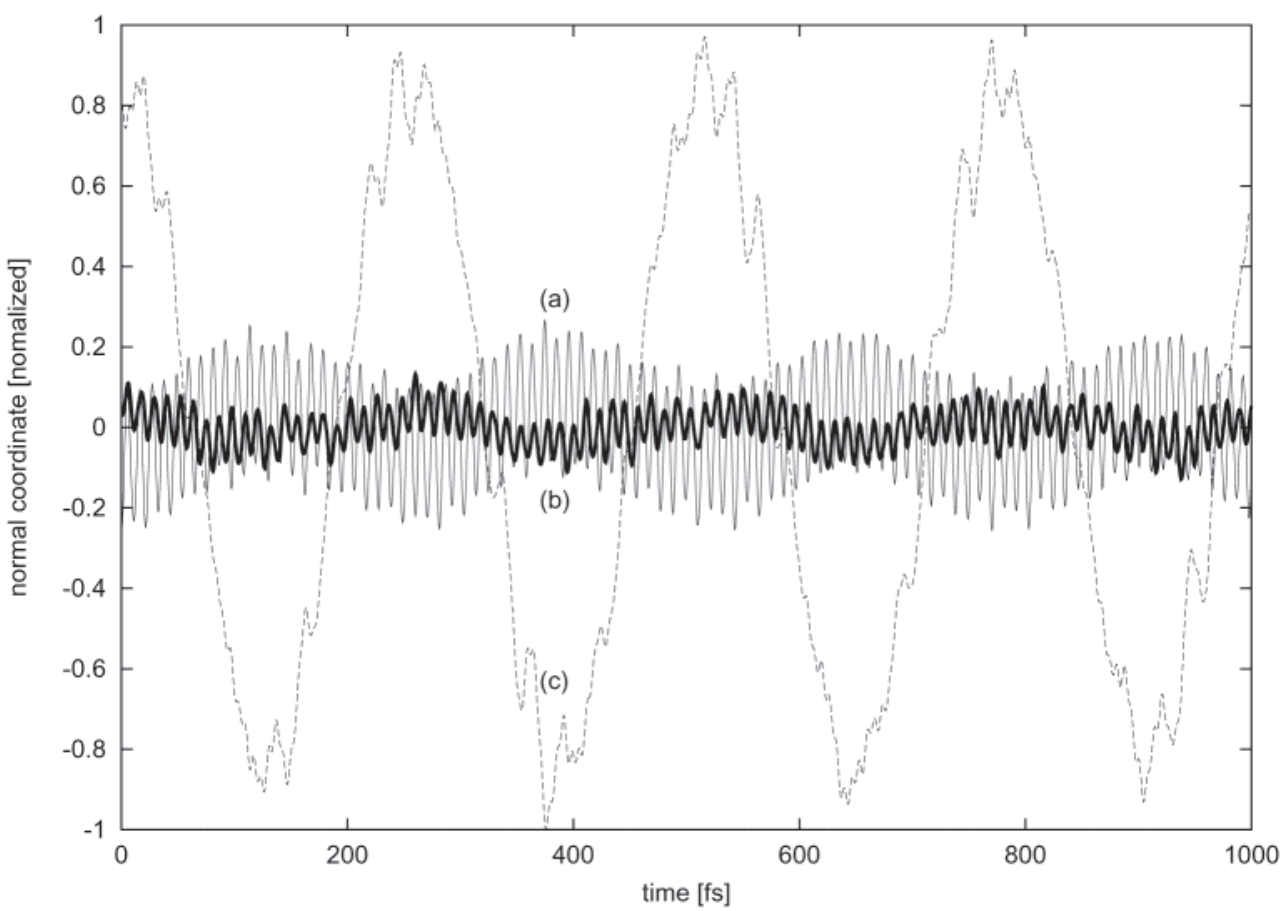

Figure 11. Upper panel: Cartesian displacements corresponding to the excited-state Raman active vibrational modes of the PDA oligomer (see text). The vibrational frequencies of the modes are $v_{\mathrm{a}}=3080 \mathrm{~cm}^{-1}, v_{\mathrm{b}}=2893 \mathrm{~cm}^{-1}$, and $v_{\mathrm{c}}=130 \mathrm{~cm}^{-1}$. These frequencies are off with respect to the experimental values, ${ }^{24}$ especially for the high-frequency $\mathrm{C} \equiv \mathrm{C}$ vibrations, assigned to a peak located at $2080 \mathrm{~cm}^{-1}$. This overestimate of the vibrational frequencies is a spurious effect due to the particular parametrization of the ZINDO Hamiltonian, which has been used for describing the ground-state potential surface (see text). Lower panel: time evolution of the displacements along the excited-state vibrational eigenvectors of the PDA, as discussed in the text.

Liouville space matrix element:

$$
\Omega_{v}=\left(\xi_{v}|L| \xi_{v}\right)
$$

Taking the derivative with respect to a generic nuclear coordinate $x$, we get

$$
\Omega_{v}^{x}=\left(\xi_{v}^{x}|L| \xi_{v}\right)+\left(\xi_{v}|L| \xi_{v}^{x}\right)+\left(\xi_{v}\left|L^{x}\right| \xi_{v}\right)
$$

The hermiticity of the Liouville operator with respect to our scalar product makes it possible to act with $L$ both to the right and to the left, obtaining the following for the first two terms of eq A2: $\Omega_{v}\left[\left(\xi_{v}^{x} \mid \xi_{v}\right)+\left(\xi_{v} \mid \xi_{v}^{x}\right)\right]=0$. This term vanishes because of the condition on the derivative of the normalization of the modes. The excitation energy gradient is finally given by eq 16 .

\section{References and Notes}

(1) Gussoni, M.; Castiglioni, C.; Zerbi, G. In Vibrational Spectroscopy of New Materials; Clark, R. J. H., Hester, R. E., Eds.; Wiley: New York, 1991.

(2) Castiglioni, C.; Del Zoppo, M.; Zerbi, G. J. Raman Spectrosc. 1993, $24,485-494$ 
(3) Castiglioni, C.; Del Zoppo, M.; Zerbi, G. Phys. Rev. B 1996, 53 $13319-13325$.

(4) Del Zoppo, M.; Castiglioni, C.; Zuliani, P.; Zerbi, G. In Handbook of Conducting Polymers; Skotheim, T. A., Elsenbaumer, R. L., Reynolds, J., Eds.; Dekker: New York, 1998.

(5) Zerbi, G.; Gussoni, M.; Castiglioni, C. In Conjugated Polymers: Novel Science and Technology of Conducting and Non Linear Optically Active Materials; Bredas, J. L., Silbey, J., Eds.; Kluwer: New York, 1991; pp 436-507. 5230.

6) Lasaga, A. C.; Aerni, R. J.; Karplus, M. J. Chem. Phys. 1980, 73,

(7) Zerbetto, F.; Zgierski, M. Z.; Negri, F.; Orlandi, G. J. Chem. Phys.

1988, 89, 3681 .

(8) Negri, F.; Orlandi, G.; Zerbetto, F.; Zgierski, M. Z. J. Chem. Phys. 1989, 91,6215 .

(9) Del Zoppo, M.; Tommasini, M.; Castiglioni, C.; Zerbi, G. Chem. Phys. Lett. 1998, 287, 100-108.

(10) Tang, J.; Albrecht, A. C. In Raman Spectroscopy; Szymanski, H., Ed.; Plenum: New York, 1970; Vol. 2.

(11) Inagaki, F.; Tasumi, M.; Miyazawa, T. J. Raman Spectrosc. 1975, 3,335

(12) (a) Fincher, C. R.; Ozaki, M.; Tanaka, M.; Peebles, D.; Lauchlan, L.; Heeger, A. J.; MacDiarmid, A. G. Phys. Rev. B 1979, 20, 1589. (b) Shirakawa, H.; Ito, T.; Ikeda, S. Polym. J. 1973, 4, 460.

(13) Soos, Z. G.; Mukhopadhyay, D.; Painelli, A.; Girlando, A. In Handbook of Conducting Polymers; Skotheim, T. A., Elsenbaumer, R. L., Reynolds, J., Eds.; Dekker: New York, 1998.

(14) Castiglioni, C.; Gussoni, M.; Del Zoppo, M.; Zerbi, G. Solid State Commun. 1992, 82, 13.

(15) Castiglioni, C.; Tommasini, M.; Del Zoppo, M. J. Mol. Struct. 2000, $521,137$.

(16) Tommasini, M.; Castiglioni, C.; Zerbi, G. J. Mol. Struct. (THEOCHEM) 2000, 500, 323.

(17) Del Zoppo, M.; Castiglioni, C.; Tommasini, M.; Mondini, P.; Magnoni, C.; Zerbi, G. Synth. Met. 1999, 102, 1582

(18) Verlet, L. Phys. Rev. 1967, 159, 98; 165, 201

(19) Tsiper, E. V.; Chernyak, V.; Tretiak, S.; Mukamel, S. J. Chem. Phys. 1999, 110, 8328

(20) Chernyak, V.; Mukamel, S. J. Chem. Phys. 1996, 104, 444

(21) Mukamel, S.; Tretiak, S.; Wagersreiter, T.; Chernyak, V. Science 1997, 277, 781 .

(22) Tretiak, S.; Chernyak, V.; Mukamel, S. J. Am. Chem. Soc. 1997, 119,11408
(23) Tretiak, S.; Chernyak, V.; Mukamel, S. Int. J. Quantum Chem. 1998, 70,711

(24) Kobayashi, T.; Shirakawa, A.; Matsuzawa, H.; Nakanishi, H. Chem. Phys. Lett. 2000, 321, 385.

(25) Domcke, W.; Stock, G. Adv. Chem. Phys. 1997, 100, 1.

(26) Avery, J. Creation and Annihilation AOperators; McGraw-Hill: New York, 1976.

(27) Feynman, R. P. Phys. Rev. 1939, 56, 340

(28) Chernyak, V.; Schulz, M. F.; Mukamel, S.; Tretiak, S.; Tsiper, E. V. J. Chem. Phys. 2000, 113, 36.

(29) (a) Lehoucq, R. B.; Maschhoff, K. J.; Sorensen, D. C.; Yang, C. (http://www.caam.rice.edu/software/ARPACK/download, accessed 4/20/2000) (b) Lehoucq, R. B. Ph.D. Thesis, Rice University, Houston, Texas, 1995. Technical Report TR95-13; Dept. of Computational and Applied Mathematics, Rice University: Houston, Texas, 1995. (c) Maschhoff, K. J.; Sorensen, D. C. P_ARPACK: An Efficient Portable Large Scale Eigenvalue Package for Distributed Memory Parallel Architectures. In Applied Parallel Computing in Industrial Problems and Optimization; Wasniewski, J., Dongarra,

J., Madsen, K., Olesen, D., Eds.; Lecture Notes in Computer Science; Springer-Verlag: Berlin, 1996; Vol. 1184.

(30) Frenkel, D.; Smit, B. Understanding Molecular Simulation; Academic Press: New York, 1996.

(31) Thompson, M. A.; Zerner, M. C. J. Am. Chem. Soc. 1991, 113, 8210. Thompson, M. A.; Glendening, E. D.; Feller, D. J. Phys. Chem. 1994 98, 10465. Thompson, M. A.; Schenter, G. K. J. Phys. Chem. 1995, 99 6374. Thompson, M. A. J. Phys. Chem. 1996, 100, 14492. For INDO references, see: Ridley, J.; Zerner, M. TCA (Berlin) 1973, 32, 111. Zerner, M. C.; Loew, G. H.; Kirchner, R. F.; Mueller-Westerhoff, U. T. J. Am Chem. Soc. 1980, 102, 589. Zerner, M. C. Semiempirical Molecular Orbital Methods. In Reviews in Computational Chemistry II; Libkowitz, K. B., Boyd, D. B., Eds.; VCH Publishers: New York, 1991; Chapter 8, p 313

(32) Turki, M.; Barisien, T.; Bigot, J. Y.; Daniel, C. J. Chem. Phys. 2000, 112, 10526

(33) Karabunarliev, S.; Baumgarten, M.; Bittner, E. R.; Müllen, K. J. Chem. Phys. 2000, 113, 11372 .

(34) Wilson, E. B.; Decius, J. C.; Cross, P. C. Molecular Vibrations; McGraw-Hill: New YOrk, 1955

(35) Mizushima, S; Shimanouchi, T. J Chem Phys 1967, 47, 3605. Krimm, S. Ind. Pure Appl. Phys. 1978, 16, 335. Frazer, G. V. Ind. Pure Appl. Phys. 1978, 16, 344.

(36) Chernyak, V. Mukamel, S. J. Chem. Phys. 2000, 112, 3572.

(37) Tommasini, M.; Chernyak, V.; Mukamel, S. Int. J. Q. Phys. 2001, (In Press). 